\title{
Testing discrepancy effects: A critique, a suggestion, and an illustration
}

\author{
MIRON ZUCKERMAN \\ University of Rochester, Rochester, New York \\ MARYLÈNE GAGNÉ \\ Concordia University, Montreal, Quebec, Canada \\ IRIS NAFSHI \\ University of Rochester, Rochester, New York \\ and \\ C. RAYMOND KNEE and SUZANNE C. KIEFFER \\ University of Houston, Houston, Texas
}

\begin{abstract}
To assess the effects of discrepancy between two independent variables, investigators sometimes compute difference scores and correlate such scores with a criterion variable. However, the correlation of the difference with the criterion is accounted for by the correlations of the difference constituents with the criterion and the constituents' variances. It follows that when investigators are testing a prediction that is not captured by the difference constituents' main effects, using the difference correlation analysis may be misleading. Under these circumstances, the effects of a discrepancy between two independent variables can be assessed by a test of their interaction. The problems inherent in using difference scores and the advantage of testing the interaction are illustrated in relation to research programs on two separate topics in social psychology.
\end{abstract}

Researchers sometimes use the difference between the scores of two independent variables, $a$ and $b$, as a predictor of the criterion variable(s). Instances of this practice can be classified into two distinct categories. In the first category, researchers assume that $a$ and $b$ represent the same theoretical construct, and they view the difference between $a$ and $b$ as a better representation of that construct than is either $a$ or $b$ alone. ${ }^{1}$ In the second category, researchers do not assume that $a$ and $b$ represent the same construct. More important, they view the difference between $a$ and $b$ as an operational definition of a new construct, one that is not represented by either $a$ or $b$.

In the present paper, we propose that usings difference scores when $a$ and $b$ (and their difference) represent the same construct is legitimate. In contrast, using difference scores when $a$ and $b$ (and their difference) represent different constructs may lead us astray. To clarify this distinction, we first illustrate the "legitimate" practice of difference scores analysis.

An instance of difference scores representing the same construct as that represented by $a$ and $b$ is the resultant achievementmotivation (RAM). RAM is calculated as the

M.G. was supported by a doctoral fellowship from the Social Sciences and Humanities Research Council of Canada. Correspondence concerning this article should be addressed to M. Zuckerman, Department of Clinical and Social Sciences in Psychology, University of Rochester, Rochester, NY 14627 (e-mail: miron@ @sych.rochester.edu). difference between standardized need-for-achievement scores (operationalized via a projective measure of $n$ achievement) and standardized fear-of-failure scores (operationalized via a measure of test anxiety; Atkinson \& Litwin, 1960; see also Atkinson \& Feather, 1966). In this procedure, need for achievement and fear of failure are viewed as opposites, with the former facilitating and the latter inhibiting achievement behavior. Stated differently, need for achievement, fear of failure, and their difference are all viewed as representatives of the same construct, but the difference score is seen as its best operational definition. A similar procedure is sometimes used to classify people as autonomous or control oriented (Deci \& Ryan, 1991). Autonomous individuals are said to regulate their behavior according to inner needs; control-oriented individualsare said to regulate their behavior according to environmental controls. Behavior regulation is often measured by Deci and Ryan's (1985) General Causality Orientation Scale, which includes separate autonomy and control orientation subscales. Koestner, Bernieri, and Zuckerman (1992; see also Koestner \& Zuckerman, 1994) classified experimental participants as either autonomous or control oriented, depending on which of the two subscales yielded higher standardized scores. Underlying this classification is the assumption that autonomy and control orientation are two opposites of a single construct and that the difference between the two subscales better represents that construct than does either subscale alone. 
Not surprisingly, critiques of this use of difference scores analysis target its theoretical underpinning. Specifically, researchers may argue that $a$ and $b$ do not represent the same construct and, hence, should not be combined (by subtraction). Researchers have argued, for example, that need for achievement and fear of failure should be treated as two independent variables, rather than as opposites of a single construct (Covington, 1992; Elliot, 1997). Similar arguments have been put forth to halt the practice of combining some other well-known pairs of variables via their difference scores (e.g., masculinity and femininity, negative and positive affect).

The reason investigators calculate difference scores that represent the same construct as do $a$ and $b$ is that the difference scores pack more predictive power than do their constituents. For example, RAM was viewed as a better predictor than either need for achievement or fear of failure. The reason for calculating difference scores that represent a construct that $a$ and $b$ do not represent is to test predictions that $a$ and $b$ cannot test. For example, investigators often use difference scores to test the implications of the similarity-discrepancy between self-evaluation(variable $a$ ) and some standard (variable $b$ ). Such implications cannot be tested by either self-evaluation or the standard alone.

In calculating difference scores that are meant to represent a new construct, researchers have followed one of two procedures, which we term here as simple difference and residual difference. In the simple-difference procedure, $a$ and $b$ are often made equivalent (e.g., by conversion to standard scores), and then one is subtracted from the other. In the residual-difference procedure, $a$ (usually self-evaluation) is regressed on $b$ (usually the standard), allowing the investigators to compute the residual difference, $d$ ( $a$ minus the $a$ predicted from $b$ ). Either the simple difference or the residual difference is then used to predict the dependent variable.

The problem with simple difference scores is that they are completely predictable from $a$ and $b$. Consequently, it is impossible to examine the relation between the difference scores and the criterion while controlling for the effects of $a$ and $b$. When $a$ is controlled for, the simple difference will have the same relation as $b$ with the dependent variable (and vice versa). If $a$ and $b$ are not controlled for, the difference correlation with the criterion reflects the main effect correlations of $a$ and $b$ with the criterion. This is not a problem if the difference scores are meant to represent the same construct as $a$ and $b$. It is a problem if the difference scores are meant to represent a new construct and, therefore, to be used in testing predictions that $a$ and $b$ cannot test.

The residual-difference analysis was introduced as an improvement over the simple-difference procedure, but it has its own share of problems. The correlation of the residual difference of $a$ with the criterion is the same as the correlation of $a$ with the criterion, controlling for $b$. Controlling for $b$ equates all individuals on $b$, so that the resulting partial correlation of $a$ with the criterion cannot test different hypotheses regarding the implications of the $a-b$ discrepancy. Indeed, when $a$ and $b$ are uncorrelated, the residual difference is identical to $a$ and is completely divorced from the concept of the $a-b$ discrepancy. Finally, note that it is impossible to correlate the residual difference of $a$ with the criterion $y$, controlling for $a$, because this correlation equals the correlation of $b$ with $y$, controlling for $a$, except that the sign is reversed: $r_{d(y \bullet a)}=$ $-1 \times r_{b(y \bullet a)}$. (For a more detailed critique of the residualdifference procedure, see Assor, Tzelgov, Thein, Ilardi, \& Connell, 1990; Griffin, Murray, \& Gonzalez, 1999).

It should be noted that experimental designs with pretest and posttest measures of the dependent variable lead to analyses that use either the simple or the residual difference. The simple-difference procedure is used in an analysis of variance with pre-post change scores as the dependent variable. The residual-difference procedure is used in an analysis of covariance of posttest scores with the pretest scores as the covariate or in a regression analysis in which pretest scores are entered as the first predictor of posttest scores, to be followed by other predictors of interest. Investigators typically do not worry about the relation of change scores to the pre- and posttest measures. Indeed, the current scientific paradigm is such that investigators worry more about lack of independence among independent variables than about that among dependent variables. Consequently, the current critique applies to situations in which simple and residual differences are used as independent variables, but not as dependent variables.

Is it possible to test the effects of the discrepancy between $a$ and $b$ independently of the effects of $a$ and $b$ ? We propose that this task can be accomplished by a test of the $a \times b$ interaction, conducted via an analysis of variance or a regression analysis. Using a hypothetical example, we will illustrate why the simple difference does not test the discrepancy effects, whereas the interaction does. Suppose that an investigator wants to test the hypothesis that a discrepancy between low self-evaluation and a high standard is associated with poorer health. Using the simple-difference method, the investigator decides to subtract (after standardization) a measure of self-evaluation from a measure of the standard and to correlate the difference with a measure of health. The investigator will interpret a negative difference correlation as support for the hypothesis. ${ }^{2}$

Two problems arise. The first one is less relevant to the present point but is included for the sake of complete presentation. The negative difference correlation gives rise to several interpretations. It may reflect a positive correlation of self-evaluation with health, a negative correlation of the standard with health, or both. ${ }^{3}$ Termed the confounding of difference scores with their constituents (Edwards, 1994; Griff in et al., 1999; Johns, 1981), this problem cannot be resolved unless the investigator looks at the constituents' main effect correlations. Importantly, we want to know which of the two variables (self-evaluation or the standard) drives the difference correlation, because these two variables represent two different constructs.

The first problem leads directly to the second. Insofar as the difference correlation is a function of the constituents' correlations (and their variance, unless they are standard- 
ized), its interpretation also depends on the constituents' correlations. In the present example, the negative difference correlation may indicate that (1) lower self-esteem is related to poorer health, if that is the constituent correlation that drives the difference correlation, (2) a higher standard is related to poorer health, if that is the constituent correlation that drives the difference correlation, or (3) both are related to poorer health. All three interpretations fail to address the hypothesis that the discrepancy between self-esteem and the standard is related to poorer health. Stated differently, if the hypothesis does not focus on the main effects of self-evaluation and/or the standard, the simple-difference procedure cannot test it. In contrast, the self-evaluation $\times$ standard interaction indicates whether, in addition to their main effects, self-evaluation and the standard together influence health. One set of hypothetical results may show that lower self-esteem is related to poorer health (main effect), that a higher standard is related to poorer health (main effect), and that the combination of low self-esteem and a high standard is related to an additional decline in health (interaction effect). This interaction effect, which is completely independent of the main effects, will support the investigator's hypothesis.

In the remainder of this paper, we will present several studies in which difference scores have been used to examine the effects of the discrepancy between $a$ and $b$. Following a critique of these studies, we will present two sets of data and a test of the interaction as an alternative method of analyzing discrepancy effects. In the first data set, the interaction is tested via an analysis of variance; in the second set, the interaction is tested via a regression analysis.

\section{DISCREPANCY AS A MEASURE OF SELF-ENHANCEMENT}

\section{The Colvin, Block, and Funder (1995) Study}

In a highly influential article, Taylor and Brown (1988) suggested that the tendency to engage in self-enhancementthat is, overly positive self-evaluation - is related to better psychological adjustment. Colvin and Block (1994) argued, however, that the existing data lend little support for the benefits of positive self-enhancement. Colvin, Block, and Funder (1995) conducted three experiments in order to further examine the issue. In all three experiments, the investigators assessed the simple difference between participants' self-evaluation (termed the self-favorability index) and others' evaluation (termed the other-favorability index) of the participants. The self-favorability index was calculated for each participant as the correlation between the participants' self-rating on a Q-sort and judges' favorability ratings of each of the Q-sort adjectives (higher correlations imply that the participants were more likely to describe themselves with adjectives that the judges considered favorable). The other-favorability index was calculated for each participant as the correlation between observers' ratings of the participants on a Q-sort and judges' favorability ratings of each of the Q-sort adjectives. For each participant, the other-favorabilityindex was subtracted from the self-favorability index (Fisher $r-z$ transformations were used before the subtraction), resulting in a selfenhancement score. The higher the self-enhancement score, the more favorably the participants evaluated themselves, relative to evaluations by the observers.

In two experiments, the self-enhancement scores were correlated with personality ratings provided by trained examiners and friends 5 years before or 5 years after the assessment of self-enhancement. In the third experiment, the self-enhancement scores were correlated with the participants' behavior in a debate, coded by trained observers. In all three experiments, higher self-enhancement was related to ratings reflecting poor social skills and psychological maladjustment.

In a critique of these findings, Zuckerman and Knee (1996) argued that in all three of Colvin et al.'s (1995) experiments, the effect of self-enhancement was confounded with those of the self- and other-favorability indices. The solution, according to Zuckerman and Knee, is to replace the simple difference procedure with a test of the interaction. In their response, Colvin, Block, and Funder (1996) argued that the use of a simple difference is justified if it is an appropriate definition of the construct. Given that the construct is self-enhancement, the difference between self- and other-favorability indices provides "a direct, easily understandable, and readily analyzable index" of that concept (p. 1253). They also maintained that a test of the interaction between self- and other-favorability is equivalent to a test of the residual difference (regressing selffavorability on other-favorability, calculating the residual difference, and correlating that difference with the dependent variable). They rejected the residual difference procedure as too problematic.

Zuckerman and Knee's (1996) critique was not very specific. They did not provide an interpretation of Colvin et al.'s (1995) results, and they did not show how the interaction can actually test the self-enhancement effect. These shortcomings are even more pronounced in view of Colvin et al.'s (1996) response. To address these issues, we will first present the formula for a correlation between a simple difference score and a criterion $y, r_{y d}$. According to J. Cohen and P. Cohen (1983, p. 416),

$$
r_{y d}=r_{y(a-b)}=\frac{r_{y a} s d_{a}-r_{y b} s d_{b}}{\sqrt{s d_{a}^{2}+s d_{b}^{2}-2 r_{a b} s d_{a} s d_{b}}} .
$$

When $a$ and $b$ are standardized, $s d=1$, and the equation can be simplified to

$$
r_{y d}=r_{y(a-b)}=\frac{r_{y a}-r_{y b}}{\sqrt{2-2 r_{a b}}} .
$$

In their response, Colvin et al. (1996) provided some of the correlations that make up Equations 1 and 2. Correlations of self-favorability with the criterion $\left(r_{y a}\right)$ ranged from .31 to .47 , with a median of .42 ; correlations of otherfavorability with the criterion $\left(r_{y b}\right)$ ranged from .42 to .56 , with a median of .48 ; the difference correlations ranged from -.23 to -.33 , with a median of -.24 . As Equations 1 and 2 indicate, the reason that the simple difference was 
negatively correlated with the criterion is that otherfavorability was more highly correlated with the criterion than was self-favorability. In all likelihood, the reason for the higher correlations of other-favorability with the criterion is that both other-favorability and the criterion were based on observers' ratings of the participants. If the criterion were based on some form of self-rating, one might expect higher correlations of self-favorability with the criterion and, in accordance with Equations 1 and 2, positive correlations between self-enhancement and the criterion.

Colvin et al.'s (1995) results thus lead to two very different interpretations. The interpretation favored by Colvin et al. (1995) is that overly positive self-evaluation is maladaptive, whereas accurate self-evaluation is adaptive. ${ }^{4}$ The alternative interpretation is that the negative correlation of the criterion with self-enhancement reflects higher correlations of the criterion with other-favorability than with self-favorability, a consequence of a design in which both the criterion and other-favorability were based on observers' ratings of the participants.

As was previously noted, Colvin et al. (1996) also argued that a test of the interaction is equivalent to the problematic residual difference procedure (see also Asendorpf \& Ostendorf's [1998, p. 956] concurrence with this position). But the residual difference procedure and the interaction are very different analyses. The $a \times b$ interaction is completely independent of the main effects of both $a$ and $b$; the correlation of $a$ 's residual difference with a criterion represents $a$ 's main effect, controlling for $b$.

\section{The Asendorpf and Ostendorf (1998) Study}

To further examine correlates and psychometric properties of self-enhancement, Asendorpf and Ostendorf (1998) conducted an experiment in which participants rated themselves on a large number of traits and were each rated by three acquaintances on the same traits. For each trait, the investigators calculated three self-enhancement scores as the differences between a participant's self-rating and each of the three acquaintances' ratings. Each of these three difference scores was correlated with acquaintances' ratings that were not a part of it (e.g., the difference in ratings between self and Acquaintance 1 was correlated with ratings by Acquaintance 2 and again with ratings by Acquaintance 3 ). The three self-enhancement scores thus generated six self-enhancement correlations. These correlations were averaged, yielding a mean self-enhancement correlation for each trait. Overall, the self-enhancement correlations tended to be negative, particularly for the more evaluative traits (traits judged as more favorable or unfavorable). Importantly, the data also showed that otherother correlations were greater than self-other correlations, particularly for more evaluative traits.

The experimental design and results were similar to those of Colvin et al. (1995). Like Colvin et al. (1995), Asendorpf and Ostendorf (1998) measured self-enhancement as the difference between self- and other-ratings and operationalized the criterion on the basis of other-ratings. Once again, the results lead to two very different inter- pretations. One interpretation is that self-enhancement is linked to unfavorable traits and, therefore, is maladaptive. The alternative interpretation is that the simple difference correlation is a function of the constituents' main effect correlations (and their variances). Specifically, because otherother correlations were higher than self-other correlations, the difference between self- and other-ratings (i.e., self-enhancement) was also negatively correlated with other-ratings. If the criteria had been based on self-ratings, the results would have been in the opposite direction.

\section{Testing Discrepancy Effects via the Interaction}

The present study, part of a larger project, was longitudinal in nature, with a 2-month duration. We examined whether discrepancy measured at Time 1 predicted adjustment at Time 2, controlling for adjustment at Time 1. Discrepancy was derived from measures of self- and otherfavorability; adjustment was assessed by self-report measures of health and mood.

\section{Method}

Two hundred and fifteen college undergraduates (127 women and 88 men) participated in the study in partial fulfillment of an introductory course requirement. The participants filled out a battery of personality questionnaires at the beginning (Time 1) and again at the end (Time 2) of the fall semester. At both times, the participants completed the questionnaires in small groups over a period of 2 weeks. At Time 1, each participant provided the names of two roommates, who were then contacted by phone and asked to provide an assessment of the participant's personality. Those who agreed were met with individually by the experimenter and were administered a personality scale. In all, 186 participants were each rated by two roommates, and 29 participants were rated by a single roommate. The roommates of 49 additional participants could not be contacted or refused to participate; these participants were not included in the present study.

Self- and peer-ratings. The participants rated themselves and were rated by their roommates on a 50 -item adjective inventory. The adjectives were selected to represent the "Big Five" personality structure from a list provided by McCrae and Costa (1987). Each of the five factors was represented by 10 adjectives. The adjectives were randomly ordered in the scale but were blocked for factor so that each group of five adjectives represented all five factors. The rating scale for each adjective ranged from 1 (not at all characteristic) to 7 (very characteristic). For participants who were rated by two roommates, we calculated average ratings (interrater reliability $=$ .71), so that each participant now had a single peer-rating profile.

Health measures. The participants completed the General Health Questionnaire (GHQ; Goldberg \& Hillier, 1979) and the CohenHoberman Inventory of Physical Symptoms (CHIPS; F. Cohen \& Hoberman, 1983). The GHQ is a 28 -item measure of psychosomatic symptoms (e.g., "lost much sleep over worry"). The rating scale for each item ranges from 1 (not at all) to 4 (much more than usual). The CHIPS is a list of 36 physical ailments (e.g., back pains, nosebleeds) that excludes symptoms of a psychological nature (e.g., depression). The rating scale for each item ranges from 0 (not at all) to 4 (extremely). At both Time 1 and Time 2, the participants rated the GHQ and CHIPS symptoms on the extent to which they experienced them during the previous 3 weeks. Because GHQ and CHIPS scores were highly correlated (.66 at Time 1 and .71 at Time 2), they were transformed into standard scores and were summed to form a single health measure. Higher scores on this measure indicated poorer health.

Mood. The participants filled out the two 10-adjective mood scales that make up the Positive and Negative Affect Schedule 
Table 1

Predicting Health and Mood from Self-Favorability, Other-Favorability, and Simple Difference Scores

\begin{tabular}{|c|c|c|c|}
\hline & Self & Other & $\begin{array}{c}\text { Simple } \\
\text { Difference }\end{array}$ \\
\hline \multicolumn{4}{|c|}{ Concurrent Analysis } \\
\hline Health & $-.45^{* * *}$ & -.09 & $-.24 * * *$ \\
\hline PANAS NA & $-.53 * * *$ & $-.16^{*}$ & $-.23 * *$ \\
\hline PANAS PA & $.49 * * *$ & .09 & $.26 * * *$ \\
\hline \multicolumn{4}{|c|}{ Longitudinal Analysis } \\
\hline Health & -.10 & .13 & $-.17^{*}$ \\
\hline PANAS NA & $-.19^{*}$ & -.04 & -.08 \\
\hline PANAS PA & $.16^{*}$ & -.09 & $.18^{*}$ \\
\hline
\end{tabular}

Note-The concurrent analysis includes partial correlations, controlling for sex. The longitudinal analysis includes partial correlations, controlling for sex and the appropriate baseline measure. PANAS, Positive and Negative Affect Scale. NA and PA indicate negative and positive mood, respectively. ${ }^{*} p<.05 .{ }^{* *} p<.01 .{ }^{* * * *} p<.001$.

(PANAS; Watson, Clark, \& Tellegen, 1988). At Time 1, participants rated how they felt generally, whereas at Time 2, the participants indicated how they felt during the past 3 weeks. The rating scale for each item ranged from 1 (very slightly or not at all) to 7 (extremely). The scale yielded two scores, one (PANAS PA) representing positive mood and one (PANAS NA) representing negative mood.

\section{Results and Discussion}

Self- and other-favorability indices were formed in accordance with the procedure used by Colvin et al. (1995). First, a group of 20 undergraduate judges rated each of the 50 adjectives that were used in the self-ratings and the peer ratings on a favorability scale. For each adjective, the judges rated, on a scale of 1 (very unfavorably) to 7 (very favorably), "How favorably or unfavorably would you regard a person who possessed this trait?" (Colvin et al., 1995 , p. 1154). Interjudge reliability, computed from the 20 judges $\times 50$ adjectives matrix, was high (Cronbach alpha $=.97)$. Accordingly, judges' ratings were aggregated to form a 50-item favorability profile. We then computed (1) the self-favorability index by calculating a Pearson correlation between each participant's 50 self-ratings and the judges' 50-item favorability profile and (2) the other-favorability index by calculating a Pearson correlation between each participant's 50 peer ratings and the judges' 50 -item favorability profile. For both self- and otherfavorability, higher correlations indicated more positive evaluations. The other-favorability index was subtracted from self-favorability (after Fisher $r-z$ transformation), yielding a self-enhancement score for each participant.

It was expected that the health and mood criteria would be more strongly related to self-favorability than to otherfavorability and, therefore, that self-enhancement (selffavorability minus other-favorability) would be positively correlated with better health and mood (see Equations 1 and 2). To examine this prediction, we conducted one concurrent correlational analysis and a second, longitudinal correlational analyses. In the concurrent analysis, we correlated the two favorability indices and their difference (the self-enhancement score) with the three criteria (selfreported health, PANAS NA, and PANAS PA) from Time 1, partialling out sex. In the longitudinal analysis, we correlated the indices and their difference with the three criteria from Time 2, partialling out sex and the appropriate baseline from Time 1 . As can be seen in Table 1, selffavorability was more strongly related to better health and mood than was other-favorability. Accordingly, selfenhancement was also related to better health and moodthe exact opposite of the results reported by Colvin et al. (1995) and Asendorpf and Ostendorf (1998).

Self-enhancement effects are more appropriately measured via a test of the interaction. Accordingly, we classified the participants into low, medium, and high levels on both self-favorability and other-favorability. Their reported health and PANAS NA and PA scores from Time 2 were examined in three separate $3 \times 3 \times 2$ (self-favorability $\times$ other-favorability $\times$ sex $)$ analyses of covariance, with reported health or the PANAS NA or PA scores from Time 1 as the covariates. Tables 2 and 3 present mean scores and interaction residuals for reported health and PANAS NA from Time 2. (The PANAS PA did not reveal any result of interest and will not be discussed further.) Table 4 presents relevant effects from the analyses. It can be seen that lower levels of self-favorability resulted in poorer reported health and more negative affect (linear contrasts, $p$ s $<$ $.05)$. Higher levels of other-favorability resulted in poorer reported health (linear contrast, $p<.05$ ), but not in more negative mood.

More relevant to the issue at hand, the residual interaction scores in Table 2 indicate that the decrease in reported health owing to lower self-favorability was less emphasized in the low other-favorability condition (residuals decline from .250 to -.145 ) and was more emphasized in the high other-favorability condition (residuals increase from -.203 to .209). Stated differently, in addition to and completely independent of the two main effects, overly negative self-evaluation (low self-favorability and high other-favorability) produced particularly poor reported health. However, the interaction between the self- and the

Table 2

Reported Health Scores by Self-Favorability and Other-Favorability Levels

\begin{tabular}{lcccc}
\hline \multirow{5}{*}{ Other-Favorability } & \multicolumn{4}{c}{ Self-Favorability } \\
\cline { 2 - 5 } & Low & Medium & High & Mean \\
\hline \multicolumn{5}{c}{ Raw Scores } \\
Low & -.193 & -.437 & -.233 & -.288 \\
Medium & .009 & -.092 & -.405 & -.163 \\
High & .640 & .143 & -.205 & .193 \\
Mean & .152 & -.129 & -.281 & -.086 \\
& Interaction Residuals & & \\
Low & -.143 & -.106 & .250 & 0 \\
Medium & -.066 & .114 & -.047 & 0 \\
High & .209 & -.007 & -.203 & 0 \\
Mean & 0 & 0 & 0 & 0 \\
\hline
\end{tabular}

Note-Higher scores reflect poorer health. 
Table 3

PANAS NA Scores by Self-Favorability and Other-Favorability Levels

\begin{tabular}{lcccc}
\hline \multirow{5}{*}{ Other-Favorability } & \multicolumn{4}{c}{ Self-Favorability } \\
\cline { 2 - 5 } & Low & Medium & High & Mean \\
\hline \multicolumn{4}{c}{ Raw Scores } \\
Low & 26.7 & 23.9 & 26.5 & 25.7 \\
Medium & 24.8 & 24.2 & 21.4 & 23.5 \\
High & 29.8 & 26.0 & 22.6 & 26.1 \\
Mean & 27.1 & 24.7 & 23.5 & 25.1 \\
& Interaction & Residuals & & \\
Low & -1.00 & -1.40 & 2.40 & 0 \\
Medium & -0.71 & 1.16 & -0.46 & 0 \\
High & 1.70 & 0.25 & -1.94 & 0 \\
Mean & 0 & 0 & 0 & 0 \\
\hline
\end{tabular}

Note-Higher scores reflect more negative affect. PANAS NA, Positive and Negative Affect Scale-Negative Affect.

other-favorability linear contrasts was not significant $(p<$ .12). On the other hand, the means at the top of Table 2 indicate a synergistic pattern such that the combination of low self- and high other-favorability resulted in poorer reported health $(M=.640)$, relative to all other combinations (means ranging from -.437 to +.143 ). Indeed, the contrast between low-self-favorability/high-other-favorability and all the other cells was significant $[F(1,196)=$ $8.07, p<.01]$; the residual $F$, reflecting variance not accounted for by the synergistic contrast, was small $[F(7,196)=0.47]$.

The interaction residuals of the PANAS NA (see the bottom half of Table 3 ) show a very similar pattern. This time, the interaction between self- and other-favorability linear contrasts was significant $(p<.05)$. The comparison between the low-self-favorability/high-other-favorability cell $(M=29.8)$ and all the other cells (means ranging from 21.4 to 26.7$)$ was significant $[F(1,165)=9.01, p<$ $.01]$; the residual $F$ was not $[F(7,165)=1.20(d f$ s for the PANAS NA are lower than $d f_{\text {s }}$ for reported health, because the PANAS was added to the study after it already had started)].

The analysis above was meant to illustrate how discrepancy effects can be examined via a test of the interaction. The actual results are not conclusive. The interaction between the linear contrasts of self- and other-favorability was significant for the PANAS NA, but not for self-reported health. The synergistic contrasts were significant for both variables, but the one for the PANAS NA was accompanied by a residual $F$ that was large enough to suggest that other significant contrasts may exist in the data (the maximum $F$ for any other contrast is obtained by the product of the residual $F$ and its $d f_{\mathrm{s}}-1.20 \times 7=8.40$ for the PANAS NA, but only $0.47 \times 7=3.29$ for reported health). 5

Note that if other-favorability plays the role of the "standard" in our previous example, the results lend some support to the discrepancy hypothesis of our hypothetical investigator. They show that in addition to their main effects, a low self-evaluation (low self-favorability) combined with high standard (high other-favorability) led to poorer health and more negative mood. Perhaps when others' expectations are high (as indicated by high other-favorability) but confidence in one's own potential is low (as indicated by low self-favorability), feelings of inadequacy or helplessness result, accompanied perhaps by anxiety over disappointing one's peers and friends. This perspective is consistent with the classic definition of stress (Lazarus \& Folkman, 1984) as demands that exceed one's resources and potential. When self-favorability is lower than otherfavorability, the individuals in question may believe that they cannot live up to the standards set for them by friends and peers. To repeat, these conclusions should be treated with caution.

\section{DISCREPANCY AS A MEASURE OF PRONENESS TO PREJUDICED RESPONSES}

A body of research indicates that many people who claim that they are not prejudiced manifest prejudice in their actual behavior. This discrepancy is particularly evident when the self-reported low-prejudice attitudes are compared with automatic or nonconsciously monitored behavior (e.g., Dovidio, Kawakami, Johnson, Johnson, \& Howard, 1997; Gilbert \& Hixon, 1991). The discrepancy led Devine (1989) to propose a distinctionbetween automatic prejudicelike responses and controlled nonprejudiced behavior. The distinction thus allows for the coexistence of nonprejudiced beliefs and prejudiced thoughts within the same person. Furthermore, Devine's model suggests that many people are aware of the inconsistency between their beliefs and their behavior and are open to direct questions about it. Indeed, several studies have shown that many people report that they show greater prejudice in their behavior than what their own personal standards would justify (e.g., Devine, Monteith, Zuwerink, \& Elliot, 1991; Monteith, 1996a, 1996b; Monteith, Devine, \& Zuwerink, 1993). In these studies, participants indicated, for five different situations involving stereotyped groups, the extent to which they should have a prejudiced response and the extent to which they would have such a response. The difference between the should and would responses served as an index of prejudice-related discrepancies.

Table 4

Effects of Self-Favorability and Other-Favorability on Reported Health and PANAS NA Scores

\begin{tabular}{lcc}
\hline \multicolumn{1}{c}{ Effect } & $\begin{array}{c}\text { Reported Health } \\
(F)\end{array}$ & $\begin{array}{c}\text { PANAS NA } \\
(F)\end{array}$ \\
\hline Linear self-favorability & $3.96^{*}$ & $7.19^{* *}$ \\
Linear other-favorability & $4.93^{*}$ & 0.12 \\
$a \times b$ & $2.28^{+}$ & $4.70^{*}$ \\
Synergistic contrast & $8.07^{*}$ & $9.01 * *$ \\
Residual effect & 0.47 & 1.20 \\
\hline
\end{tabular}

Note- $d f_{\text {error }}$ was 196 for perceived health and 165 for PANAS NA (Positive and Negative Affect Scale-Negative Affect). ${ }^{+} p<.12 . \quad * p<.05$. $* * p<.01$. 


\section{The Monteith and Voils (1998) Study}

Recently, Monteith and Voils (1998) developed a more extensive 32-item discrepancy questionnaire, consisting of 16 should items (i.e., ones requiring participants to state how they should respond in situations involving Blacks) and 16 would items (i.e., ones requiring participants to state how they actually would respond in the same 16 situations). Higher scores on both the should and the would portions of the questionnaires indicate higher levels of prejudice. Using the simple difference procedure, discrepancy scores were created by subtracting the should scores from the would scores, so that a higher discrepancy score indicates that a participant's self-reported behavior (would scores) shows greater prejudice than the participant self-reported standards (should scores). The investigators then examined personality, affective, and behavioral correlates of the discrepancy scores. In general, the results supported the construct validity of the scale. For example, higher discrepancy scores were related to self-report of more negative affect (e.g., discomfort and self-criticism). Interestingly, Monteith and Voils mentioned (p. 904, note 3) that they repeated all analyses, using the residual difference procedure (i.e., regressing would on should scores, calculating the residual scores, and correlating the residual scores with criterion variables). The results were essentially the same as those obtained by the simple-difference procedure.

Note that the should scores represent personal standards, the would scores represent actual behavior, and the simple difference between them was meant to represent a third construct (the extent to which one is not living up to one's standards). Because the simple difference correlation reflects the constituents' main effect correlations (and their variances), it reflects some combination of the should and the would main effects. It is not really testing the discrepancy effect, because the should and the would main effects do not test the discrepancy effect.

\section{Testing Discrepancy Effects via the Interaction}

As an illustration of a more appropriate test of discrepancy effects, the present experiment was designed to reex- amine a small portion of the findings reported by Monteith and Voils (1998). Specifically, we tested the relation of discrepancy to three personality constructs and three affective responses. The personality constructs were private and public self-consciousness and social anxiety (Fenigstein, Scheier, \& Buss, 1975); the affective responses were negative feelings directed toward the self (termed negself), general uneasiness (termed discomfort), and feelings of well-being (termed positive; see Devine et al., 1991).

\section{Method}

Two hundred and nine Caucasian college undergraduates (142 women and 66 men; 1 participant did not identify his or her gender) participated in the study in return for extra credit. The participants filled out a battery of questionnaires in small groups. Three questionnaires, to be described below, belonged to the present project; the rest were part of another, unrelated study.

Discrepancy questionnaire. The participants completed the 32item measure constructed by Monteith and Voils (1998). The first 16 items described how the participants believed they should respond to Blacks (e.g., "I believe I should not think of Blacks in stereotypical ways"). The next 16 items described how the participants believed they would respond to Blacks (e.g., "I sometimes have stereotypical racial thoughts"), with each item corresponding in content to one of the should items appearing in the first part. For each of the 32 items, the participants rated their level of agreement on a 7-point scale (1, strongly agree; 7, strongly disagree). Items were reverse-scored when necessary, so that higher scores always indicated higher prejudice. Reliabilities (Cronbach alphas) of the should and the would indices were .78 and .86 , respectively; as could be expected, reliability of the discrepancy indices (would minus should) was somewhat lower $(\alpha=.67)$.

Self-consciousness. The participants completed the Fenigstein et al. (1975) Public and Private Self-Consciousness Scale, which yields scores for public self-consciousness $(\alpha=.76)$, private selfconsciousness $(\alpha=.67)$, and social anxiety $(\alpha=.78)$.

Affective responses. The participants were presented with a list of 21 affect items (Devine et al., 1991, Experiment 2) that consisted of three subscales: negself (e.g., disappointed with myself), discomfort (e.g., fearful), and positive (e.g., friendly). The instructions informed the participants that we were interested in their feelings about the consistency or disparity between their should and their would responses to the discrepancy questionnaire. For each item, the participants rated the extent to which the particular affect described their feeling on a 7-point scale (1, does not apply at all; 7, applies very

Table 5

Correlations of Self-Consciousness and Affect Measures With the Should, Would, and Simple-Difference Indices

\begin{tabular}{|c|c|c|c|c|c|c|}
\hline \multirow[b]{2}{*}{ Scale } & \multicolumn{2}{|c|}{ Should Index } & \multicolumn{2}{|c|}{ Would Index } & \multicolumn{2}{|c|}{ Simple-Difference Index } \\
\hline & $\begin{array}{c}\text { Monteith and } \\
\text { Voils (1998) }\end{array}$ & $\begin{array}{c}\text { Present } \\
\text { Study }\end{array}$ & $\begin{array}{c}\text { Monteith and } \\
\text { Voils (1998) }\end{array}$ & $\begin{array}{c}\text { Present } \\
\text { Study }\end{array}$ & $\begin{array}{c}\text { Monteith and } \\
\text { Voils (1998) }\end{array}$ & $\begin{array}{c}\text { Present } \\
\text { Study }\end{array}$ \\
\hline \multicolumn{7}{|l|}{ Self-consciousness } \\
\hline Private self-consciousness & -.13 & -.08 & -.01 & .06 & .12 & $.16^{*}$ \\
\hline Public self-consciousness & .13 & .04 & $.32 * *$ & $.25 * *$ & $.36^{* *}$ & $.30 * *$ \\
\hline Social anxiety & .12 & .05 & $.28 * *$ & $.29 * *$ & $.30 * *$ & $.35 * *$ \\
\hline \multicolumn{7}{|l|}{ Affect } \\
\hline Discomfort & - & $.25 * *$ & - & $.37 * *$ & $.39 * * *$ & $.28 * *$ \\
\hline Negself & - & .10 & - & $.27 * *$ & $.43 * * *$ & $.28 * *$ \\
\hline Positive & - & $-.15^{*}$ & - & $-.36^{* *}$ & n.s. & $-.36 * *$ \\
\hline
\end{tabular}

Note-For the Monteith and Voils study, the top half of the table presents zero-order correlations of the selfconsciousness scales with the should, would, and simple-difference indices (Monteith \& Voils, 1998, Experiment 1), and the bottom half of the table presents partial correlations of the affective subscales with the simple-difference index, holding prejudice constant (Monteith \& Voils, 1998, Experiment 2). For the present study, zero-order correlations are presented throughout. $* p<.05$. $* * p<.01$. $* * * p<.001$. 
much). Ratings on relevant items were averaged, thus yielding scores on negself $(\alpha=.93)$, discomfort $(\alpha=.89)$, and positive $(\alpha=.91)$.

\section{Results and Discussion}

We first compared the results of a correlational analysis conducted by Monteith and Voils (1998) with a similar analysis of the present data. The top half of Table 5 presents zero-order correlations of private and public selfconsciousness and social anxiety with the should, the would, and the simple-difference indices. Two correlations are presented in each cell, one from Monteith and Voils (1998, Experiment 1) and one from the present study. It can be seen that in both studies, the simple difference (would minus should) correlated positively with all three self-consciousness scales, although the correlations are somewhat lower for private self-consciousness. It can also be seen that in both studies, the simple difference correlations are driven by the higher correlations of the would (as compared with the should) index with the selfconsciousness scales. Accordingly, the simple-difference correlations give rise to two different interpretations. The interpretation espoused by Monteith and Voils is that higher discrepancy (being more prejudiced then one should) is related to higher public self-consciousness and higher social anxiety. The alternative interpretation is that the simpledifference correlations are largely due to the would main effect correlations, which indicate that being more prejudiced is related to higher public self-consciousness and higher social anxiety. These two interpretations appear very similar, but the one focusing on the would main effect is more parsimonious and corresponds better to the actual results.

The bottom half of Table 5 presents data from Monteith and Voils (1998) and from the present investigation that are only roughly comparable. Monteith and Voils (1998, Experiment 2) used the simple-difference index together with the Attitude Toward Blacks (ATB) scale (Brigham, 1993 ) to predict each of the three affect subscales: discomfort, negself, and positive. Their analysis, therefore, yields partial correlations of the simple-difference index with the three affects, holding ATB constant (the would and the should indices were not used in this analysis, and hence, no results were reported concerning their relations with the affect subscales). The present study did not include the ATB, and hence, we report the comparable zeroorder correlations. It can be seen that in both studies, the simple difference was positively correlated with discomfort and negself. In the present study, the simple difference was also negatively correlated with positive, whereas Monteith and Voils reported this condition as nonsignificant (the actual $r$ was not included). Finally, the data from the present study indicate that the single-difference correlations are driven by the higher correlations of the would (as compared with the should) index with all three affects. Once again, therefore, the difference correlation reflects the constituents' main effect correlations, rather than the discrepancy effect.

A more appropriate test of discrepancy effects is the test of the would $\times$ should interaction. This test was conducted via six separate regression analyses in which the three self-consciousness scales and the three affect subscales served as dependent variables. First, each of the three self-consciousness scales was regressed on the should and the would indices (entered simultaneously as predictors in Step 1) and on the product of the should and the would indices (entered as a predictor in Step 2). The would $\times$ should product (representing linear $\times$ linear interaction) was the appropriate predictor because we intended to examine whether high levels on the would index (representing more prejudiced responses) combined with lower levels on the should index (representing less prejudiced standards) would be related to particularly high levels on each of the "dependent" variables. Sex, originally entered into the regression equation in Step 1, was not related to any of the three self-consciousness scales and was subsequently dropped from the analyses. These analyses were repeated for the three affect subscales, except that, this time, sex was related to both discomfort and negself and was thus included in all three analyses.

A summary of the results is presented in Table 6. For each dependent variable, the table presents the partial correlation of the predictor with the dependent variable and

Table 6

Predicting Self-Consciousness and Affect from Indices of Should, Would, and Should $\times$ Would Interactions

\begin{tabular}{|c|c|c|c|}
\hline Scale & Should Index & Would Index & $\begin{array}{c}\text { Should } \times \text { Would } \\
\text { Interaction }\end{array}$ \\
\hline \multicolumn{4}{|l|}{ Self-consciousness } \\
\hline Private self-consciousness & $-.17 *$ & $.16^{*}$ & -.04 \\
\hline Public self-consciousness & $-.18 * *$ & $.30 * * *$ & $-.16^{*}$ \\
\hline Social anxiety & $-.22 * *$ & $.35 * * *$ & -.02 \\
\hline \multicolumn{4}{|l|}{ Affect } \\
\hline Discomfort & .03 & $.30 * * *$ & -.09 \\
\hline Negself & -.08 & $.30 * * *$ & -.08 \\
\hline Positive & .13 & $-.37 * * *$ & $.15^{*}$ \\
\hline $\begin{array}{l}\text { Note-For the self-conscious } \\
1,205 \text { for the interaction; for th } \\
1,203 \text { for the interaction (sex } \\
\text { of } 1 d f \text {; a missing value for se } \\
* * * p<.001 .\end{array}$ & $\begin{array}{l}\text { subscales, } d f \\
\text { fect subscales, } \\
\text { added to the ar } \\
\text { used a loss of }\end{array}$ & $\begin{array}{l}\text { ere } 1,206 \text { for } \\
\text { were } 1,204 \text { fo } \\
\text { ses involving } \\
\text { her } 1 d f) \text {. }\end{array}$ & $\begin{array}{l}\text { main effects and } \\
\text { e main effects and } \\
\text { ect, causing a loss } \\
.05 . \quad * * p<.01 .\end{array}$ \\
\hline
\end{tabular}



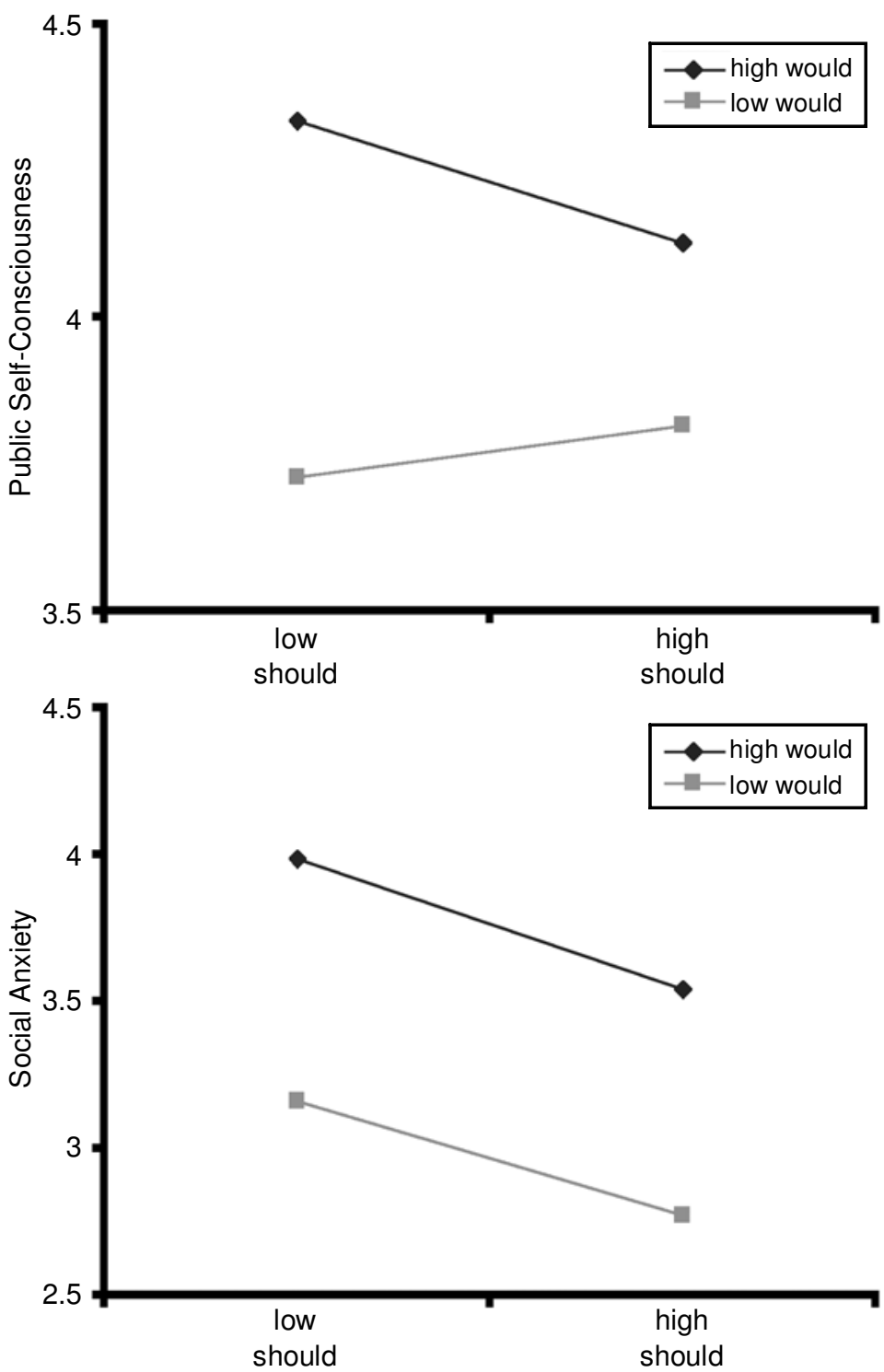

Figure 1. Predicted values for public self-consciousness and social anxiety as a function of should and would scores.

its significance level. It can be seen that each of the three self-consciousness scales was negatively related with the should index and positively related with the would index. The would $\times$ should interaction was significant for public self-consciousness, but not for the two other scales. To illustrate the one significant interaction and, for comparison purposes, one nonsignificant interaction, we used the regression equations to identify predicted public selfconsciousness and social anxiety scores for high and low levels of the predictors (cf. J. Cohen \& P. Cohen, 1983, chap. 8). Scores of both variables were thus calculated for high and low levels (one standard deviation above and one standard deviation below the mean, respectively) of both the should and the would indices. Figure 1 presents the results.
The results for public self-consciousness provide support for the predicted discrepancy effect. Specifically, public self-consciousness was greater for higher levels of the would index, but the increase was more pronounced for lower levels of the should index. Thus, high would scores (indicating prejudiced responses) combined with low should scores (indicating low standards for prejudice) were associated with particularly high public selfconsciousness. In contrast, the results for social anxiety do not show discrepancy effects. Social anxiety was greater for higher levels of would and lower levels of should scores, but the combination of high would and low should scores did not produce additional increase in social anxiety.

Turning to the three affects, higher would scores were related to more negative (higher discomfort and negself) 



Figure 2. Predicted values for the positive and negself affects as a function of should and would scores.

and less positive affect, the should index was not related to any affect, and the would $\times$ should interaction was significant only for the positive affect. Using the procedure described above, the one significant interaction for positive and the one nonsignificant interaction (for negself) are illustrated in Figure 2. The results support the predicted discrepancy effect for positive affect and were in the predicted direction for negself. The pattern of interaction for discomfort was similar to that of negself, but as is noted in Table 6, neither interaction was significant. An additional regression analysis in which the dependent variable was a combination of negself and discomfort (they were highly correlated, $r=.84$ ) also failed to show a significant would $\times$ should interaction $[F(1,203)=1.69, p=.19]$.

Given that the interaction and the simple-difference correlation yield different results, the investigator must choose one of the two as a basis for conclusions. For example, is social anxiety related to the discrepancy between would and should? According to the simple-difference correlation, it is (see Table 5, right-hand column). However, the regression analysis showed only two main effects (see Figure 1), which account for the simple-difference correlation without having to explain it in terms of discrepancy. As a result of the two main effects, the participants high on would and low on should were in fact high on social anxiety. But the parsimonious explanation is that the finding reflects would and should main effects without adding discrepancy to the mixture. Turning to public self-consciousness, the simple-difference correlation indicates that the participants high on would and low on should were, in fact, high on public self-consciousness, but once again this result can be fully accounted for by the 
would and the should main effects. This time, however, there was also an interaction effect, which indicates that in addition to the main effects, the combination of high would and low should produced a further increase in public self-consciousness. This further increase is an indication of a discrepancy effect.

\section{CONCLUSIONS}

The present paper could be retitled as "why investigators should not trust their common sense in data analysis." There is nothing more appealing or more straightforward than using the simple-difference procedure to examine discrepancy effects. At the same time, there is nothing appealing or straightforward in the suggestion that the effect of a discrepancy between two variables should be examined via their interaction. Nevertheless, the major thesis of the present paper is that a test of interaction is a more appropriate procedure for testing discrepancy effects than is the simple-difference procedure.

It is important to reiterate the limiting conditions of the present proposition. Investigators often combine variables by subtraction or addition because the combined variable has more predictive power than its constituents. Typically, the combined variable (such as a simple difference) represents the same construct as the constituents-this is the rationale for combining them in the first place. For example, an investigator may compute excess masculinity scores by subtracting femininity from masculinity (the current view that femininity and masculinity are independent notwithstanding). In these circumstances, it is quite appropriate that the simple-difference effects are accounted for by the effects of the difference constituents. In fact, it was the investigator's intent to capitalize on the two main effects via the difference procedure.

Suppose, however, that the simple difference is meant to measure a new construct and to test predictions that cannot be tested by the difference constituents. Thus, otherfavorability was subtracted from self-favorability to measure self-enhancement (Colvin et al., 1995). Once again, the simple-difference effect is accounted for by the constituents' effects, although now the simple difference was meant to test predictions that could not be tested by its constituents. The constituents' correlations with a criterion that was based on other-ratings showed greater otherother agreement than self-other agreement, and this produced a negative difference correlation with the same criterion. Stated differently, self-enhancement appeared maladaptive because other-other correlations were higher than self-other correlations. As is shown by the present data, using a criterion that was based on self-ratings led to a positive difference correlation with the criterion. Stated differently, self-enhancement appeared adaptive because selfself correlations were higher than self-other correlations.

In our other example, should (personal norms about prejudice) was subtracted from would (actual behavior prejudice) to test predictions about discrepancy effects (Monteith \& Voils, 1998). The problem here is more sub- tle but no less real. The would and should measures are not opposites of the same construct, and neither represents the construct of discrepancy. As Table 5 indicates, the correlations of the simple difference with the criteria (selfconsciousness scales and the affective scales) reflect stronger correlations of the would (relative to the should) with these criteria. Why would rather than should was related to the criteria is not instructive about discrepancy effects; yet these differential relations account for the simpledifference correlations which are meant to test discrepancy effect. This point parallels the previous critique about using simple difference to test self-enhancement. In both cases, main effects that do not test the effects of a particular construct account for the simple-difference effect that is meant to test that very same construct.

We proposed that the interaction test does assess discrepancy effects and illustrated its use via an analysis of variance for one data set and via a regression analysis for another. We will comment only briefly here about the merits of the two procedures, since this issue is familiar to most readers. In general, an analysis of variance is more likely to be used with categorical variables than with continuous data. The reason is that categorizing continuous data results in some loss of information. On the other hand, some interaction contrasts (including the synergistic contrast that was tested here) require that continuous variables be categorized.

It is important to note that the interaction test is a solution for the specific problem we raised here, but not for other, unrelated problems. A case in point is the use of the (absolute) difference score as a gage of self-other agreement or consistency of self-ratings. Self-other agreement could be measured by the absolute difference between self-ratings and other-ratings (of the self); consistency, or self-self agreement, could be measured by the absolute difference between self-ratings on two or more occasions. Self-other agreement typically measures accuracy in the perception of a person. However, Bem and Allen (1974) conceptualized self-other agreement and self-self agreement as two alternative measures of cross-situational consistency and, hence, degree of "traitedness." In all these instances, the difference scores represent a variable that is not represented by the difference constituents. Is a test of the interaction appropriate here? The answer is that neither the simple (absolute) difference procedure nor the interaction test is appropriate.

In a well known critique, Cronbach and Gage (Cronbach, 1955; Gage \& Cronbach, 1955) showed that self-other differences represent no fewer than four components. To illustrate one component, high self-other agreement can represent stereotype accuracy - that is, a tendency by all people to rate a particular trait higher (or lower) relative to other traits. Note that although the critique was directed at self-other difference scores, it is equally valid in relation to self-self difference scores. Note also that the critique is applicable to interaction effects. Inasmuch as self-other difference may reflect any combination of the four components, a self $\times$ other interaction may do the same. For 
example, an interaction showing that participants high in self-other agreement scores differ from participants low in self-other agreement scores may reflect the effect of stereotype accuracy. In other words, Gage and Cronbach's critique makes both difference scores and interaction effects difficult to interpret. For remedial procedures that yield more exact measures of self-other (and by extension self-self) agreement, see Bernieri, Zuckerman, Koestner, and Rosenthal, 1994.

Even when interaction tests are appropriate, they do have their own share of problems. Given the importance attributed here to the distinction between main effects and interaction, investigators must be cautious about any transformation (e.g., log transformation) that may change the balance between the two. Investigators also may find that the two variables involved in the interaction are related, and even a weak or moderate relation can create problems (e.g., small number of participants in some cells and loss of power). Treatment of this and other issues in testing interactions can be found in several recent publications: See Abelson and Prentice (1997) and Rosenthal, Rosnow, and Rubin (2000) for a discussion of interaction and contrast testing in analysis of variance; and see Aiken and West (1991) for a comparable discussion of interaction in regression analysis.

\section{REFERENCES}

Abelson, R. P., \& Prentice, D. A. (1997). Contrast tests of interaction hypotheses. Psychological Methods, 2, 315-328.

AIKEN, L. S., \& WEST, S. G. (1991).Multiple regression: Testing and interpreting interactions. Newbury Park, CA: Sage.

AsENDORPF, J. B., \& OSTENDORF, F. (1998). Is self-enhancement healthy? Conceptual, psychometric, and empirical analysis. Journal of Personality \& Social Psychology, 74, 955-966.

Assor, A., Tzelgov, J., Thein, R., Ilardi, B. C., \& Connell, J. P. (1990). Assessing the correlates of over- and underrating of academic competence: A conceptual classification and a methodological proposal. Child Development, 61, 2085-2097.

AtKinson, J. W., \& Feather, N. T. (EDS.) (1966). A theory of achievement motivation. New York: Wiley.

AtKinson, J. W., \& Litwin, G. H. (1960). Achievement motive and test anxiety conceived as motive to approach success and motive to avoid failure. Journal of Abnormal \& Social Psychology, 60, 52-64.

BEM, D. J., \& ALLEN, A. (1974). On predicting some of the people some of the time: The search for cross-situational consistencies in behavior. Psychological Review, 81, 506-520.

Bernieri, F., Zuckerman, M., Koestner, R. \& Rosenthal, R. (1994) Measuring person perception accuracy: Another look at self-other agreement. Personality \& Social Psychology Bulletin, 20, 367-378

BrighaM, J. C. (1993). College students' racial attitudes. Journal of Applied Social Psychology, 23, 1933-1967.

Cohen, F., \& Hoberman, H. (1983). Positive events and social support as buffers of life change stress. Journal of Applied Social Psychology, 13, 99-125.

Cohen, J., \& Cohen, P. (1983). Applied multiple regression/correlation analysis for the behavioral sciences (3rd ed.). Hillsdale, NJ: Erlbaum.

Colvin, C. R, \& Block, J. (1994). Do positive illusions foster mental health? An examination of the Taylor and Brown formulation. Psychological Bulletin, 116, 3-20.

Colvin, C. R, Block, J., \& Funder, D. C. (1995). Overly positive selfevaluation and personality: Negative implications for mental health. Journal of Personality \& Social Psychology, 68, 1152-1162.

Colvin, C. R., Block, J., \& Funder, D. C. (1996). Psychometric truths in the absence of psychological meaning: A reply to Zuckerman and Knee. Journal of Personality \& Social Psychology, 70, 1252-1255.
Covington, M. W. (1992). Making the grade: A self-worth perspective on motivation and school reform. New York: Cambridge University Press.

CRONBACH, L. J. (1955). Processes affecting scores on "underestimating of others" and "assumed similarity." Psychological Bulletin, 52, 177193.

Deci, E. L., \& Ryan, R. M. (1985). The General Causality Orientations Scale: Self-determination in personality. Journal of Research in Personality, 19, 109-134.

Deci, E. L., \& Ryan, R. M. (1991). A motivational approach to self: Integration in personality. In R. Dienstbier (Ed.), Nebraska symposium on motivation (Vol. 38, pp. 237-288). Lincoln: University of Nebraska Press.

Devine, P. G. (1989). Stereotypes and prejudice: Their automatic and controlled opponents. Journal of Personality \& Social Psychology, 56, 5-18.

Devine, P. G., Monteith, M. J., Zuwerink, J. R. \& Elliot, A. J. (1991). Prejudice with and without compunction. Journal of Personality \& Social Psychology, 60, 817-830.

Dovidio, J. F., KaWaKami, K., Johnson, C., Johnson, B., \& Howard, A (1997). On the nature of prejudice: Automatic and controlled processes. Journal of Experimental Social Psychology, 33, 510-540.

EDWARDS, J. R. (1994). The study of congruence in organizational behavior research: Critique and a proposed alternative. Organizational Behavior \& Human Decision Processes, 58, 51-100.

ElLIOT, A. J. (1997). Integrating the "classic" and "contemporary" approaches to achievement motivation: A hierarchical model of approach and avoidance achievement motivation. In M. Maehr \& P. Pintrich (Eds.), Advances in motivation and achievement (Vol. 10, pp. 143-179). Greenwich, CT: JAI Press.

Fenigstein, A., Scheier, M. F., \& Buss, A. H. (1975). Public and private self-consciousness: Assessment and theory. Journal of Consulting \& Clinical Psychology, 43, 522-527.

Gage, N. L., \& Cronbach, L. J. (1955). Conceptual and methodological problems in person-perception. Psychological Review, 62, 411422 .

Gilbert, D., \& Hixon, J. G. (1991). The trouble with thinking: Activation and application of stereotypic beliefs. Journal of Personality \& Social Psychology, 60, 509-517.

Goldberg, D. P., \& Hillier, V. F. (1979). A scaled version of the General Health Questionnaire. Psychological Medicine, 9, 139-145.

Griffin, D., Murray, S., \& GonZalez, R. (1999). Difference score correlations in relationship research: A conceptual primer. Personal Relationships, 6, 505-518.

JoHNS, G. (1981). Difference score measures of organizational behavior variables: A critique. Organizational Behavior \& Human Performance, 27, 443-463.

Koestner, R., Bernieri,F., \& ZuCKerman,M. (1992). Self-determination and consistency between attitudes, traits, and behaviors. Personality \& Social Psychology Bulletin, 18, 52-59.

Koestner, R, \& ZuCKerman, M. (1994). Causality orientations, failure, and achievement. Journal of Personality, 62, 321-346.

Lazarus, R. S., \& Folkman, S. (1984). Stress, appraisal, and coping. New York: Springer-Verlag.

McCrae, R. R., \& Costa, P. T., JR. (1987). Validation of the five-factor model of personality across instruments and observers. Journal of Personality \& Social Psychology, 52, 81-90.

Monteith, M. J. (1996a). Affective reactions to prejudice-related discrepant responses: The impact of standard salience. Personality \& Social Psychology Bulletin, 22, 48-59.

Monteith, M. J. (1996b). Contemporary forms of prejudice-related conflict: In search of a nutshell. Personality \& Social Psychology Bulletin, 22, 461-473.

Monteit h, M. J., Devine, P. G., \& Zuwerink, J. R. (1993). Self-directed versus other-directed affect as a consequence of prejudice-related discrepancies. Journal of Personality \& Social Psychology, 64, 198-210.

Monteith, M. J., \& VoILS, C. I. (1998). Proneness to prejudiced responses: Toward understanding the authenticity of self-reported discrepancies. Journal of Personality \& Social Psychology, 75, 901-916.

Rosenthal, R., Rosnow, R. L., \& Rubin, D. B. (2000). Contrasts and effect sizes in behavioral research. New York: Cambridge University Press. 
TAYLOR, S. E., \& Brown, J. D. (1988). Illusion and well-being: A social psychological perspective on mental health. Psychological Bulletin, 103, 193-210.

Watson, D., Clark, L. A., \& Tellegen, A. (1988). Development and validation of brief measures of positive and negative affect: The PANAS Scales. Journal of Personality \& Social Psychology, 54, 1063-1070.

Zuckerman, M., \& KneE, C. R. (1996). The relation between overly positive self-evaluation and adjustment: A comment on Colvin, Block, and Funder (1995). Journal of Personality \& Social Psychology, 70, $1250-1251$

\section{NOTES}

1. Whether researchers calculate the difference between $a$ and $b$ or the sum of $a$ and $b$ depends only on the direction in which $a$ and $b$ are scored. Thus, the present analysis can be applied to instances in which one independent variable is either added to or subtracted from another.

2. Note that a negative correlation may indicate not only that low selfevaluation and high standards are linked to poorer health, but also that high self-evaluation and low standards are linked to better health. We will return to this point later in the paper (see also Griffin et al., 1999, p. 507).

3. If the two independent variables are not standardized, the negative difference correlation may also reflect difference between their variances.

4. Note that the negative relation between self-enhancement and the criterion indicates not only that overly positive self-evaluation was related to negative criterion scores, but also that overly negative self-evaluation (rather than accurate self-perception) was related to positive criterion scores. For the results to support Colvin et al.'s (1995) conclusion that accurate self-perception is adaptive, the relation between self-enhancement and the criterion should be curvilinear; the linear correlations (i.e., the correlations reported by Colvin et al., 1995) should be around 0 (see note 2 ).

5. It should be noted here that although the interaction contrast is completely independent of the main effects, the synergistic contrast is not. How is it possible to ascertain that the synergistic contrast does not simply reflect two main effects- the very problem that our analysis of discrepancy effects was meant to overcome? In a $3 \times 3$ design, the weights of the synergistic contrast are the sum of the weights of two main effect contrasts, each comparing one level with the two other levels, and the interaction between these two contrasts. If only the two main effect contrasts contribute to a significant synergistic contrast, the interaction between them will not be significant, and the pattern of the means would deviate from what is expected in a synergistic contrast (i.e., all cells except one yielding equal means). The present data are equivocal with regard to both statistics. The interactions of interest were not significant $[F(1,196)=$ 1.42 for reported health and $F(1,165)=2.47$ for negative affect]. As can be seen in the text, the residual $F$ s (which indicate whether the means deviate from the weights of the synergistic contrast) were not significant, but the one obtained for the PANAS NA may have been too high for comfort. Clearly, there is a reason for caution. On the other hand, the procedure suggested here clearly offers protective mechanisms against the charge that the findings simply reflect the sum of the two main effects.

(Manuscript received March 14, 2001; revision accepted for publication June 2, 2002.) 